\title{
A model for deriving the direct normal and diffuse horizontal irradiance from the global tilted irradiance
}

\author{
Bill Marion* \\ National Renewable Energy Laboratory, Golden, CO 80401 USA
}

* Corresponding author. Address: 15013 Denver West Parkway, Golden, CO 80401-3305, USA.

Tel: +1 3033846793. E-mail address: bill.marion@nrel.gov (B. Marion)

\begin{abstract}
A separation model was developed for deriving the direct normal irradiance (DNI) and the diffuse horizontal irradiance (DHI) from the global tilted irradiance (GTI). The model is based on the DIRINT model, but substitutes the use of the GTI in place of the global horizontal irradiance $(\mathrm{GHI})$ for determining the global clearness index $\left(K_{t}\right)$. Additionally, an iterative method is used to adjust $K_{t}$ to improve the derived values of the DNI and the DHI. The model is referred to as the GTI-DIRINT model.

When using for model input the GTI measured with a pyranometer tilted at a small angle from the horizontal $\left(10^{\circ}\right)$, the deviations between the measured DNI and DHI and the GTI-DIRINT-modeled DNI and DHI were essentially the same as those for the DIRINT model when using the GHI for model input. Increasing the tilt angle from horizontal (as great as $44^{\circ}$ for Eugene, Oregon) increased the deviations between the modeled and measured DNI and DHI, but the results were still reasonable.

The GTI-DIRINT model provides reasonable values of DNI and DHI from the GTI, and if they are subsequently used with the Perez tilted surface model to model the GTI for orientations within $30^{\circ}$ of the azimuth and tilt of the GTI used for model input, the result is as good or better than may be obtained when using a measured DNI and DHI, and without their significant instrumentation cost.
\end{abstract}

Key words: Direct and diffuse irradiance; Global tilted irradiance; Model.

\section{Introduction}

Predicting the performance of a photovoltaic (PV) system or relating its actual performance to a prediction requires accurate solar resource data. Conventional solar resource data consist of hourly or more frequent values of the global horizontal irradiance (GHI), the direct normal irradiance (DNI), and the diffuse horizontal irradiance (DHI). These irradiances are related such that the GHI equals the DHI plus the DNI multiplied by the cosine of the sun zenith angle. Consequently, if you know any two of the three irradiances, you may calculate the third. DNI and DHI values may then be used with transposition models to calculate the global tilted irradiance (GTI) incident on PV modules. Examples of transposition models are models by Perez et al. (1990), Temps and Coulson (1977), Klucher (1978), Hay and Davies (1980), Gueymard (1987), and the HDKR model (Reindl et al., 1990). Angle-of-incidence (AOI) models also use DNI and DHI values to account for losses associated with radiation reflected from the front surfaces of PV modules. Examples of AOI models are the air-glass model (Sjerps-Koomen et al., 1997) and the Sandia model (King et al., 2004).

Often, the GHI is a measured or modeled value and a separation model determines the DNI and DHI from the GHI. DNI and DHI are seldom measured with high-quality thermopile instruments, except by research institutions, because of the cost and complexity. Consequently, much work has been performed to develop separation models and to evaluate their accuracy. Engerer (2015) summarizes validation studies of separation models, with the first study occurring in the early 1980s (Spencer, 1982), and also presents his own results for existing models and three new models. Gueymard and Ruiz-Arias (2014) found 90 separation models in the scientific literature and evaluated 36 using data for arid or desert locations. Separation models have also been evaluated in combination with transposition models, such as the study by Lave et al. (2015).

This works presents a separation model for determining the DNI and DHI from the GTI. As PV deployments have progressed, PV systems with GTI measurements have increased to a significant number. Usually these measurements are performed with higher quality radiometers (compared to photo-diode radiometers used to measure the GHI, such as for agricultural networks). Consequently, a separation model using the GTI for input can potentially provide more accurate values of the DNI and DHI, from which the GHI can also be determined. These data may then be used: (a) for the input solar resource data for commercially available PV performance models that have the capability to model the performance of PV systems of any size and PV array tilt and azimuth orientations; (b) to better model the PV performance during an energy yield test for comparing the estimated 
performance to the measured yield (knowledge of the DNI permits the AOI losses to be more precisely modeled); (c) to compare the derived solar resource to the historical norms; and (d) to improve satellite-based solar irradiance data by rebalancing to remove their bias errors.

Using the GTI for input, the separation model developed under this work is a modification of the DIRINT model by Perez et al. (1992). The DIRINT model is the most frequently used model for estimating the DNI and DHI from the GHI. Compared to other models of its type, it is ranked first, or nearly so, by studies such as those performed by Ineichen (2008), Gueymard and RuizArias (2014), Engerer (2015), and Lave et al. (2015). The NREL modifications to the DIRINT model are validated using data for three climatically diverse locations: Cocoa, Florida; Eugene, Oregon; and Golden, Colorado.

\section{Modifications to DIRINT}

The DIRINT model is an improved version of the Direct Insolation Simulation Code (DISC) model developed by Maxwell (1987). The DISC model uses functional relationships between the global clearness index $\left(K_{t}\right)$, direct normal clearness index $\left(K_{n}\right)$, and air mass to model DNI using only GHI for input. The DIRINT model improves on the DISC model by (a) normalizing $K_{t}$ to a standard clear sky GHI diurnal profile, (b) using time series measurements (before-after) to better define the DNI-DHI relationship for partly cloudy conditions, and (c) allowing the optional use of dew point temperature for quantifying the effects of atmospheric precipitable water vapor.

We modified the DIRINT model to calculate the DNI and DHI from the GTI, rather than using the GHI for input values. We refer to this modified DIRINT model as the GTI-DIRINT model. One distinction between the two models is that for a tilted surface there are times during the day when the incidence angle, $\theta$, between a ray from the sun and the tilted surface normal may be greater than or equal to $90^{\circ}$; consequently, for these times the GTI measurement does not include any component of the DNI. For tilted surfaces facing the equator, these times occur early and late in the day in spring and summer because the sun rises and sets north of east and west respectively (northern hemisphere). Locations further from the equator will increase the time when $\theta$ is greater than $90^{\circ}$, as will increasing the tilt angle from horizontal. Tilted surfaces not directly facing the equator will also increase the occurrences, which then may also be present in the fall and winter. The GTI-DIRINT model solution for the DNI and DHI depends on whether $\theta$ is less than $90^{\circ}$, or $\theta$ is greater than or equal to $90^{\circ}$.

2.1 GTI-DIRINT Model for $\theta<90^{\circ}$

For times when $\theta$ is less than $90^{\circ}$, the DIRINT model was modified to use the GTI in place of the GHI, by replacing Eqn. 1 from the DIRINT model with Eqn. 2.

$$
\begin{aligned}
& K_{t}=\text { GHI } /\left[I_{0} \cdot \max \left(0.065, \cos \left(\theta_{z}\right)\right)\right] \\
& K_{t}=\text { GTI } /\left[I_{0} \cdot \max (0.065, \cos (\theta))\right]
\end{aligned}
$$

where $I_{0}$ is the extraterrestrial irradiance on a surface normal to the rays of the sun, $\mathrm{W} / \mathrm{m}^{2}$; and $\theta_{z}$ is the zenith angle between a ray from the sun and the vertical.

With $K_{t}$ determined using Eqn. 2, unmodified DIRINT equations and matrix coefficients are then used to calculate the DNI. If the GHI were known, the DHI could be determined from the GHI and DNI. But the GTI-DIRINT model uses the GTI, not the GHI, for input. Consequently, based on Eqn. 1, we use Eqn. 3 to determine the DHI.

$$
\mathrm{DHI}=K_{t} \cdot\left[I_{0} \cdot \max \left(0.065, \cos \left(\theta_{z}\right)\right)\right]-\mathrm{DNI} \cdot \cos \left(\theta_{z}\right)
$$

For small PV module tilt angles, Eqns. 1 and 2 provide similar results for $K_{t}$ (and the same results when the tilt angle is zero), but for larger PV module tilt angles the results differ because of the larger differences in the proportions of diffuse and direct irradiance for the GHI and GTI. The GTI also includes ground-reflected radiation. To compensate for errors introduced when using the GTI for input to the GTI-DIRINT model, we use the model in an iterative fashion until the measured GTI matches a modeled GTI determined by using the GTI-DIRINT-modeled values of DNI and DHI for the input to the tilted surface transposition model by Perez et al. (1990). This model is an update to the original model that was recommended by the International Energy Agency Task IX for modeling the irradiance for tilted surfaces (Hay and McKay, 1988). Fig. 1 is a flow chart showing the iterative nature of the GTI-DIRINT model for determining the DNI and DHI for $\theta<90^{\circ}$. The initial calculation uses the measured GTI to calculate $K_{t}$. Subsequent iterations adjust the GTI until the $K_{t}$ provides a DNI and DHI value such that a modeled GTI matches the measured GTI. The GTI value is adjusted according to Eqn. 4.

$$
\mathrm{GTI}_{i}=\max \left(1.0, \mathrm{GTI}_{i-1}-C \cdot D_{i-1}\right)
$$


where $i$ is the index corresponding to the iteration number; $D$ is the difference between the modeled and measured GTI, modeled minus measured, $\mathrm{W} / \mathrm{m}^{2}$; and $C$ is a coefficient to ensure an iterative solution, 1.0 for $i<3,0.5$ for $3 \leq i<10,0.25$ for $10 \leq i<20$, and 0.125 for $20 \leq i \leq 30$. Both GTI-DIRINT and the Perez tilted surface model select model coefficients from matrices and the matrix elements selected depend on the meteorological conditions. Consequently, the models are not continuous functions and this can be problematic for determining an iterative solution when using simple methods such as bisection algorithms and others that require a continuous function. The use of the $C$ coefficient, which is varied based on the iteration index, was found to be helpful. The iteration stop criteria is an absolute value of $D$ less than or equal to $1 \mathrm{~W} / \mathrm{m}^{2}$, or $i$ is greater than 30 . Often, only two iterations are needed for the solution.

Fig. 2 is the cumulative distribution function for the number of iterations when using hourly data for Eugene, Oregon, and for the GTI pyranometer tilted from the horizontal at an angle of $44^{\circ}$. As shown in Fig. 2, only 3 iterations were required for nearly $50 \%$ of the time to achieve the stop criteria of an absolute value of $D$ less than or equal to $1 \mathrm{~W} / \mathrm{m}^{2}$. The iterations were stopped about $10 \%$ of the time because the number of iterations reached the maximum allowable of 30 . When the maximum number was reached, a useful result was still provided. For the iterations that numbered 30, the average absolute value of $D$ was $2.9 \mathrm{~W} / \mathrm{m}^{2}$ (less than $1 \%$ of the average measured GTI), the standard deviation was $2.2 \mathrm{~W} / \mathrm{m}^{2}$, and the maximum was $15 \mathrm{~W} / \mathrm{m}^{2}$. In general, an increase in $\theta_{z}$ increased the number of iterations.

As discussed previously, for benefitting partly cloudy conditions, time series measurements are used to better define the DNIDHI relationship. Consequently, three measured GTI values are used with the GTI-DIRINT model: the measurement for the time of interest, and measurements immediately before and after. The measurements may be hourly averages, or represent shorter time periods such as 5 or 15 minutes. An iterative solution is determined for each time step. DIRINT was developed using hourly data, but later evaluations indicate that the performance is also acceptable, but somewhat degraded, for shorter averaging periods (Ineichen, 2008). For iterating the solution for the DNI and DHI, the before and after GTI values are also adjusted using Eqn. 4.

\subsection{GTI-DIRINT Model for $\theta \geq 90^{\circ}$}

When $\theta$ is greater than or equal to $90^{\circ}$, the GTI-DIRINT model uses an alternative method. First, using the method described in Section 2.1, the DNI and DHI values are determined for a day for all daylight times when $\theta$ is less than $90^{\circ}$. The $K_{t}^{\prime}$ values determined during the solution for the DNI and DHI are saved. $K_{t}^{\prime}$ is a $\theta_{z}$ independent expression of $K_{t}$ (Perez et al., 1992); consequently, it is more indicative of sky conditions (primarily clouds), which we assume to change slowly for this alternative method. An early morning average $K_{t}^{\prime}$ value is determined by averaging morning $K_{t}^{\prime}$ values when $\theta$ is between $65^{\circ}$ and $80^{\circ}$. A late afternoon average $K_{t}^{\prime}$ value is determined in a similar fashion. ( $K_{t}^{\prime}$ values when $\theta$ is between $80^{\circ}$ and $90^{\circ}$ are not used for the averages because of the increased error of the radiometer measurement, which negatively impacted results.) The early morning average $K_{t}^{\prime}$ value is used to determine the DNI for sunrise and later times when $\theta$ is greater than or equal to $90^{\circ}$, and the late afternoon average $K_{t}^{\prime}$ value is used to determine the DNI for sunset and earlier times when $\theta$ is greater than or equal to $90^{\circ}$.

Besides $K_{t}^{\prime}$, the solution for the DNI requires $K_{t}$, represented by Eqn. 5, which is a DIRINT equation rearranged to solve for $K_{t}$ instead of $K_{t}^{\prime}$.

$$
K_{t}=K_{t}^{\prime} \cdot(1.031 \cdot \exp (-1.4 /(0.0+9.4 / m))+0.1)
$$

where $m$ is the pressure-corrected air mass, dimensionless. Although the same average $K_{t}^{\prime}$ value is used for all times during a morning (or afternoon) when $\theta \geq 90^{\circ}$, the $K_{t}$ values, and subsequently the calculated DNI values, vary because $m$ varies with time of day. Time series measurements (before/after) are not used with this method; $K_{t}$ is only determined for the time of interest. Using the $K_{t}$ and $K_{t}^{\prime}$ values, the solution for DNI is per the DIRINT model.

When $\theta \geq 90^{\circ}$, we assume the GTI consists of diffuse radiation from an isotropic sky plus the diffuse radiation reflected from the ground, as represented by Eqn. 6 .

$$
\mathrm{GTI}=\mathrm{DHI} \cdot(1+\cos (\beta)) / 2+\left(\mathrm{DNI} \cdot \cos \left(\theta_{z}\right)+\mathrm{DHI}\right) \cdot \rho \cdot(1-\cos (\beta)) / 2
$$

where $\beta$ is the tilt angle between the surface and the horizontal; and $\rho$ is the albedo of the ground, dimensionless. Rearranging the terms provides a solution for the DHI, as represented by Eqn. 7.

$$
\mathrm{DHI}=\left[2 \cdot \mathrm{GTI}-\mathrm{DNI} \cdot \cos \left(\theta_{z}\right) \cdot \rho \cdot(1-\cos (\beta))\right] /[1+\cos (\beta)+\rho \cdot(1-\cos (\beta))]
$$




\section{Data}

The GTI-DIRINT model was evaluated using solar irradiance data for three climatically diverse locations: Cocoa, Florida (subtropical climate); Eugene, Oregon (marine west coast climate); and Golden, Colorado (semiarid climate). The data for the Cocoa and Eugene locations were measured previously as part of work to provide a publicly available data set for use in validating models that estimate the performance of flat-plate PV modules (Marion et al., 2014). The data used for this work are a subset and included the DNI, DHI, and the GTI for a tilt angle from the horizontal equaling the latitude $\left(28.5^{\circ}\right.$ for Cocoa and $44^{\circ}$ for Eugene) and south-facing. The DNI was measured with a Kipp \& Zonen CHP1 pyrheliometer, and the DHI and GTI were measured with Kipp \& Zonen CMP 22 pyranometers.

The data for Golden were new measurements and, as for Cocoa and Eugene, included the DNI, DHI, and the GTI for a tilt angle from the horizontal equaling the latitude ( $40^{\circ}$ for Golden) and south-facing. Additional GTI measurements were also performed at Golden to study the influence of the tilt and azimuth for the GTI measurement on the GTI-DIRINT model, and the use of GTIDIRINT model-derived values of DNI and DHI for modeling the GTI for other orientations. These additional GTI measurements were: south-facing with tilt angles from the horizontal of $10^{\circ}$ and $25^{\circ}$; for an azimuth of $210^{\circ}$ and tilt angle from the horizontal of $40^{\circ}$; and for two-axis tracking. The DNI was measured with a Kipp \& Zonen CHP1 pyrheliometer and the DHI was measured with a Kipp \& Zonen CMP 22 pyranometer. The GTI orientations were measured with Kipp \& Zonen CMP 11 pyranometers.

Other meteorological data for each location included the dry bulb temperature and relative humidity, which were used to determine the optional dew point temperature for input to the DIRINT and GTI-DIRINT models.

Data for one-year periods were used. This included data from March 1, 2011, through February 29, 2012, for Cocoa; data from January 1, 2013, through December 31, 2013, for Eugene; and data from April 1, 2014, through March 31, 2015, for Golden. Data were archived as 1-minute averages, and then reformatted as 5-minute, 15-minute, and hourly averages. The average or mean day-time value (using data when $\theta_{z}<90^{\circ}$ ) for each irradiance orientation and location used for the analysis are provided in Table 1. For the fixed-tilt GTI orientations, the GTI(Tilt, Orientation) notation of Vignola et al. (2012) is used where Tilt is in degrees from horizontal and Orientation is the azimuth in degrees measured eastward from true north. We designated the twoaxis tracking orientation as GTI(2-X Trkg.)

For Cocoa and Eugene, an albedo value of 0.2 (vegetation) was used when modeling the GTI. For Golden, an albedo value of 0.2 was also used for modeling the GTI, unless there was snow on the ground, in which case an albedo of 0.6 was used. Additionally, days with snow on the instruments at Golden were excluded from the analysis. This included a total of 59 days. The numbers for days excluded by month were: April 2014 (4), May 2014 (2), November 2014 (8), December 2014 (12), January 2015 (11), February 2015 (16), and March 2015 (6). For December through February, a significant number of days were excluded, but this did not seriously affect results because the days available were a reasonable representation of long-term conditions. For the days available during December through February, the average measured DNI was $4.1 \mathrm{kWh} / \mathrm{m}^{2} / \mathrm{day}$, which compares closely to the 30-year DNI average of $4.2 \mathrm{kWh} / \mathrm{m}^{2} /$ day for December through February for nearby Boulder, Colorado (Marion and Wilcox, 1994).

\section{Analysis and Results}

The performance of the GTI-DIRINT model was evaluated using the root-mean-square deviation (RMSD) and mean bias deviation (MBD) statistics, with the results expressed both in engineering units of $\mathrm{W} / \mathrm{m}^{2}$ and as a percent of the mean of the measured values from Table 1. The deviation is the measured value subtracted from the modeled value. For the MBD, a positive value indicates that the model overestimates on average. Expressions for the calculation of the MBD and the RMSD are provided as Eqns. 8 through 11.

$$
\begin{array}{ll}
\operatorname{MBD}\left(\mathrm{W} / \mathrm{m}^{2}\right) & =(1 / n) \cdot \sum\left(y_{i}-x_{i}\right) \\
\operatorname{MBD}(\%) & =100 \cdot\left[(1 / n) \cdot \sum\left(y_{i}-x_{i}\right)\right] \div\left[(1 / n) \cdot \sum x_{i}\right] \\
\operatorname{RMSD}\left(\mathrm{W} / \mathrm{m}^{2}\right) & =\left[(1 / n) \cdot \sum\left(y_{i}-x_{i}\right)^{2}\right]^{1 / 2} \\
\operatorname{RMSD}(\%) & =100 \cdot\left[(1 / n) \cdot \sum\left(y_{i}-x_{i}\right)^{2}\right]^{1 / 2} \div\left[(1 / n) \cdot \sum x_{i}\right]
\end{array}
$$

where $y_{i}$ is the $i$ th modeled value, $\mathrm{W} / \mathrm{m}^{2} ; x_{i}$ is the $i$ th measured value, $\mathrm{W} / \mathrm{m}^{2}$; and $n$ is the number of measured or modeled values.

The performance of the GTI-DIRINT model was compared to the DIRINT model for determining the DNI and DHI, and for use of the derived values of DNI and DHI to model the irradiance for other surface orientations. Comparisons were also performed 
for instances when the measured DNI and DHI are used to model the GTI. Because the use of dew point temperature is an optional input for the DIRINT model, results are presented for scenarios with and without its use. In the following sections, the analysis and results are presented for the three locations, and for the additional GTI measurements performed at the Golden location.

\subsection{Cocoa, Eugene, and Golden - Latitude-Tilt Orientation for the GTI}

For each of the three locations, 5-minute, 15-minute, and hourly averages of the GTI for a surface facing south and tilted from the horizontal at an angle equal to the location's latitude were input to the GTI-DIRINT model to model the DNI and DHI. Averages were determined as the mean measured value during the averaging period. DNI and DHI values were modeled only when $\theta_{z}<90^{\circ}$ (daytime). For 5-minute and 15-minute data, $\theta_{z}$ was determined for the time at the midpoint of the data averaging period. For hourly data, $\theta_{z}$ was also determined for the time at the midpoint (half-hour) of the data averaging period, unless a sunrise or sunset hour, in which case, $\theta_{z}$ was determined for the time at the midpoint of the period when the sun was above the horizon. For comparison with the original DIRINT model, values of the GHI were input to the DIRINT model to also model the DNI and DHI. Values of GHI were determined from the measured DNI and DHI $\left(\mathrm{GHI}=\mathrm{DNI} \cdot \cos \left(\theta_{z}\right)+\mathrm{DHI}\right)$. This approach provides the most accurate value of GHI (Gueymard, 2009).

To judge applicability when used with the Perez tilted surface model, modeled DNI and DHI values from GTI-DIRINT were used to model GHI, and modeled DNI and DHI values from DIRINT were used to model the GTI with the Perez tilted surface model. These are essentially equivalent transpositions with respect to changes in tilt angle, from GTI to GHI and from GHI to GTI. For additional comparisons, measured DNI and DHI values were also used with the Perez tilted surface model to model the GTI.

The results of the comparisons are provided in Table 2 for when using dew point temperature as a model input and in Table 3 for when dew point temperature is not used. Blanks in the table are used where the parameter was a model input and the deviation would be zero. For example, GHI is an input to DIRINT; consequently, the MBD and RMSD for modeling GHI using DNI and DHI from the DIRINT model are left blank. Similarly for the modeled GTI and the GTI-DIRINT model.

From Table 2 for when using dew point temperature as a model input, the RMSD values for DNI for the GTI-DIRINT model range from 79 to $114 \mathrm{~W} / \mathrm{m}^{2}$, and the RMSD values for DNI for the DIRINT model range from 71 to $93 \mathrm{~W} / \mathrm{m}^{2}$. Without the use of dew point temperature (Table 3), the RMSD values for DNI for the GTI-DIRINT model range from 82 to $109 \mathrm{~W} / \mathrm{m}^{2}$, and the RMSD values for DNI for the DIRINT model range from 72 to $92 \mathrm{~W} / \mathrm{m}^{2}$, which are not significantly different than when using dew point temperature. The RMSD values for DHI are about half those for DNI, with or without the use of dew point temperature.

As might be expected, the larger values are associated with a shorter averaging interval. Reducing the averaging interval from one hour to five minutes also affects the MBD values for DNI, shifting in a positive direction from $4 \%$ to $5 \%$ (4\% to $7 \%$ if not using dew point temperature), and for DHI, the MBD shifts in the opposite direction. These results are consistent with those of Ineichen (2008), who found the use of the DIRINT model for averaging intervals less than an hour acceptable, but suggested multiplicative factors be determined as a function of the averaging interval and be applied to the stability index as a means to have a consistent MBD for a different averaging interval.

However, for the purpose of modeling the GTI, this measure appears unnecessary because an error in modeled DNI is compensated to an extent by an error of the opposite sign in the modeled DHI. In Tables 2 and 3, the differences in the MBD for the GTI modeled using DIRINT-derived values of DNI and DHI for the different averaging intervals are not significant. Similarly, differences in the MBD for the GHI modeled using GTI-DIRINT-derived values of DNI and DHI for the different averaging intervals are not significant.

The MBD and RMSD results were nearly the same for: (a) using DIRINT-derived values of DNI and DHI to model the GTI, and (b) using GTI-DIRINT-derived values of DNI and DHI to model the GHI. Consequently, the models appear equivalent for transposing the irradiance from one global surface to another over the same range of tilt angles.

The use of the measured DNI and DHI for modeling the GTI provided the smallest values of RMSD—less than 4\% for hourly values. The MBD values were small—within about $\pm 0.5 \%$ from zero. The MBD values for modeling the GTI using DIRINTderived values of DNI and DHI, and the MBD values for modeling the GHI using GTI-DIRINT-derived values of DNI and DHI, were not much larger, and within the $\pm 1 \%$ bias of the pyranometer calibration.

For the GTI and GHI modeling, the RMSD results for Golden were more sensitive to the data-averaging interval. While the irradiance measurements at Cocoa and Eugene were collocated within five meters, the measurements at Golden were separated by about 330 meters. The DNI, DHI, and the GTI for the two-axis surface were measured at NREL's Solar Radiation Research Laboratory (SRRL), and the remaining GTI orientations were measured at NREL's Outdoor Test Facility. The spatial differences in measurements, due to cloud movement, become more sensitive for shorter averaging periods. For hourly averages, the effect appears less noticeable. 
Figs. 3 and 4 show hourly modeling errors for the DNI as a function of $\theta_{z}$ for Eugene, Oregon, for the DIRINT and GTIDIRINT models. In Fig. 4, data are also denoted by the GTI $\theta$ to show sensitivity of the GTI-DIRINT model to the $\theta$. Model errors are similar for both models when $\theta$ is less than $70^{\circ}$, even for large values of $\theta_{z}$, as indicated by the red plus data symbols in Fig. 4. Increased error is indicated by the blue $\mathrm{x}$ symbols $\left(70^{\circ}<\theta<90^{\circ}\right)$ and the green square symbols $\left(\theta>90^{\circ}\right)$. Fig. 5 shows the use of the GTI-DIRINT-derived hourly values of DNI and DHI to determine the hourly values of GHI for Eugene, Oregon. Errors are small for the GHI, even for the large values of the GTI $\theta$, because errors in DNI are compensated by errors of the opposite sign in DHI.

\subsection{Golden-Influence of the Tilt-Azimuth Orientation for the GTI Measurement}

The additional GTI measurements at Golden permitted evaluation of the sensitivity of the GTI-DIRINT model to the tilt and azimuth orientation of the pyranometer that provides the GTI measurements for the model. For these analyses, dew point temperatures were not input to the DIRINT and GTI-DIRINT models. The results for deriving the DNI and DHI are provided in Table 4. For the south-facing GTI measurements with a pyranometer tilt angle from the horizontal of $10^{\circ}$, the GTI-DIRINT model performance was essentially the same as the DIRINT model, with respect to both MBD and RMSD for the DNI and DHI. Larger tilt angles also performed reasonably well, with the RMSD for DNI only increasing from $18.6 \%$ to $19.3 \%$ for a tilt angle of $40^{\circ}$.

For the GTI measurements with a pyranometer tilt angle from the horizontal of $40^{\circ}$ and an azimuth of $210^{\circ}$, the performance of the GTI-DIRINT model suffered; the RMSD for DNI was almost twice as great as for the south-facing orientations. For this orientation, $\theta$ is greater than $90^{\circ}$ for about $11 \%$ of the time, and the less precise alternate GTI-DIRINT solution (Section 2.2) is used more often. However, it appears that most of the additional error occurs for $\theta$ values between $70^{\circ}$ and $90^{\circ}$. To improve GTIDIRINT for this orientation, an alternate Eqn. 2 or iteration scheme may be beneficial. South-facing orientations with the larger tilt angles from horizontal would also benefit, although degradation in their model performance was much less and occurred over a smaller range, primarily for $\theta$ 's between $80^{\circ}$ and $90^{\circ}$.

For flat-plate PV applications, the DNI and DHI are used with a transposition model to determine the GTI for a desired orientation. Fig. 6 and Fig. 7 provide MBD and RMSD values for the Perez tilted surface model when modeling the GHI and the different GTI orientations located in Golden, and when using the different model input sources of DNI and DHI (measured, DIRINT, and GTI-DIRINT). Results are shown for instances when GTI-DIRINT-derived values of DNI and DHI from each of the south-facing GTI orientations are used.

In Fig. 6, the different sources of DNI and DHI are shown essentially equivalent, with respect to MBD, for modeling the GHI and the GTI fixed-tilt orientations, with values within $1 \%$ of each other. For the GTI(2-X Trkg), the MBD values are within $2 \%$ of each other.

As shown in Fig. 7, the use of the measured DNI and DHI provided the smallest RMSD when modeling the GTI(2-X Trkg). Two-axis tracking surfaces have a large DNI component; consequently, a measured DNI with low uncertainty should provide the best result. For the fixed-tilt surfaces, the GTI-DIRINT-derived values of DNI and DHI provided the smallest RMSDs. Values of RMSD near zero are associated with the source of the data. Measured DNI and DHI were used to calculate GHI, which is also used for input to the DIRINT model. Consequently, there is no deviation when using measured DNI and DHI or DIRINTmodeled DNI and DHI to determine the GHI with the tilted surface model (tilt=0). GTI-DIRINT-modeled values of DNI and DHI also provided RMSDs near zero when used with the tilted surface model to determine the GTI for the same orientation as used for model input. Values slightly greater than zero occur because of the GTI-DIRINT model operation when $\theta$ is equal to or greater than $90^{\circ}$, which occurs more frequently with increasing tilt angle.

In Fig. 7, increasing the difference between a surface orientation for a modeled GTI and that used for the input data increased the RMSD, as shown for all sources (measured, DIRINT, or GTI-DIRINT) of DNI and DHI data. For the GTI-DIRINT results, if the tilt and azimuth for the modeled GTI is within $30^{\circ}$ of the orientation used for input to GTI-DIRINT, the use of the GTIDIRINT-derived values of DNI and DHI provide modeled GTI values with an RMSD no greater than if using measured values of DNI and DHI.

\section{Summary}

The GTI-DIRINT model was developed by modifying the DIRINT model to use input values of the GTI instead of the GHI. When the pyranometer tilt angle from horizontal was small $\left(10^{\circ}\right)$, the MBDs and RMSDs for the GTI-DIRINT-derived DNI and DHI were shown to be the same as for the DIRINT model. For the other south-facing pyranometers, increasing the tilt angle from horizontal (as great as $44^{\circ}$ for Eugene) increased the MBDs and RMSDs for the GTI-DIRINT-derived DNI and DHI, but the results were still reasonable. South-facing pyranometers were determined to be the preferred orientation; when using the GTI 
measurements for a pyranometer with a tilt angle from the horizontal of $40^{\circ}$ and an azimuth of $210^{\circ}$, the performance of the GTIDIRINT model suffered - the RMSD for DNI was almost twice as great as for the south-facing orientations.

When measured or derived values of DNI and DHI were used with the Perez tilted surface model to model the GTI, the resulting MBDs for modeling the fixed-tilt GTIs were within $1 \%$ of each other, for both the DIRINT and GTI-DIRINT model and the measured sources of data. For the two-axis tracking surface, which has a larger DNI component, the MBDs were within $2 \%$ of each other. For the GTI-DIRINT results, if the tilt and azimuth for the modeled GTI were within $30^{\circ}$ of the orientation used for input to GTI-DIRINT, the use of the GTI-DIRINT-derived values of DNI and DHI and the Perez tilted surface model provided modeled GTI values with an RMSD no greater than when using measured values of DNI and DHI. For the same locations, the DIRINT model MBDs and RMSDs for DNI are comparable to the results of a previous study by Ineichen (2008).

The GTI-DIRINT model provides reasonable values of DNI and DHI from the GTI, and if they are subsequently used with the Perez tilted surface model to model the GTI for a not-too-dissimilar orientation, the result is as good or better than may be obtained when using a measured DNI and DHI—and without their significant instrumentation cost.

\section{Acknowledgement}

This work was supported by the U.S. Department of Energy under Contract No. DE-AC36-08-GO28308 with the National Renewable Energy Laboratory (NREL). The author is thankful for the efforts of Stephen Barkaszi and Mark Jacobs (Florida Solar Energy Center) who performed the irradiance measurements in Cocoa, Florida; Frank Vignola, Rich Kessler, and Josh Peterson (University of Oregon) who performed the irradiance measurements in Eugene, Oregon; and Bill Sekulic, Jose Rodriguez, and Greg Perrin (NREL) who performed the irradiance measurements in Golden, Colorado.

\section{References}

Engerer, N., 2015. Minute resolution estimates of the diffuse fraction of global irradiance for southeastern Australia, Solar Energy 116, 215-237.

Gueymard, C., 1987. An anisotropic solar irradiance model for tilted surfaces and its comparison with selected engineering algorithms, Solar Energy 38(5), 367-386.

Gueymard, C., 2009. Direct and indirect uncertainties in the prediction of tilted irradiance for solar engineering applications, Solar Energy 83, 432-444.

Gueymard, C., Ruiz-Arias, J., 2014. Performance of separation models to predict direct irradiance at high frequency: validation over arid areas, in EuroSun 2014 Proc.

Hay, J., Davies, J., 1980. Calculation of the solar radiation incident on an inclined surface, in Proc. First Canadian Solar Radiation Data Workshop, 59-72.

Hay, J., McKay, D., 1988. Final Report IEA Task IX - Calculation of Solar Irradiances for Inclined Surfaces: Verification of Models Which Use Hourly and Daily Data, International Energy Agency Solar Heating and Cooling Program.

Ineichen, P., 2008. Comparison and validation of three global-to-beam irradiance models against ground measurements, Solar Energy 82, 501-512.

King, D., Boyson, W., Kratochvil, J., 2004. Photovoltaic Array Performance Model, Sandia National Laboratories, Albuquerque, NM.

Klucher, T., 1978. Evaluation of Models to Predict Insolation on Tilted Surfaces, National Aeronautics and Space Administration, Cleveland, $\mathrm{OH}$.

Lave, M., Hayes, W., Pohl, A., Hansen, C., 2015. Evaluation of global horizontal irradiance to plane-of-array irradiance models at locations across the United States, IEEE J. of Photovoltaics 5 (2), 597-606.

Marion, W., Wilcox, S., 1994. Solar Radiation Data Manual for Flat-Plate and Concentrating Collectors, National Renewable Energy Laboratory, Golden, CO.

Marion, B., Anderberg, A., Deline, C., del Cueto, J., Muller, M., Perrin, G., Rodriguez, J., Rummel, S., Silverman, T.J., Vignola, F., Kessler, R., Peterson, J., Barkaszi, S., Jacobs, M., Riedel, N., Pratt, L., King, B., 2014. New data set for validating PV module performance models, in $40^{\text {th }}$ IEEE PVSC.

Maxwell, E. L., 1987. A Quasi-Physical Model for Converting Hourly Global Horizontal to Direct Normal Insolation, Solar Energy Research Institute, Golden, CO.

Perez, R., Ineichen, P., Seals, R., Michalsky, J., 1990. Modeling daylight availability and irradiance components from direct and global irradiance, Solar Energy 44, 271-289.

Perez, R., Ineichen, P., Maxwell, E., Seals, R., Zelenka, A., 1992. Dynamic global-to-direct irradiance conversion models, in ASHRAE Transactions-Research Series, 354-369.

Reindl, D., Beckman, W., Duffie, J., 1990. Evaluation of hourly tilted surface radiation models, Solar Energy 45(1), 9-17. 
Spencer, J., 1982. A comparison of methods for estimating hourly diffuse solar radiation from global solar radiation, Solar Energy 29(1), 19-32.

Sjerps-Koomen, E.A., Alsema, E.A., Turkenburg, W.C., 1997. A simple model for PV module reflection losses under field conditions, Solar Energy 57, 421-432.

Temps, R., Coulson, K., 1977. Solar radiation incident upon slopes of different orientation, Solar Energy 19, 179-184.

Vignola, F., Michalsky, J., Stoffel, T., 2012. Solar and infrared radiation measurements, CRC Press, Boca Raton.

\section{Figure Captions}

Fig. 1. Flow chart for GTI-DIRINT model when $\theta<90^{\circ}$.

Fig. 2. Cumulative distribution function for the number of iterations for the GTI-DIRINT model when using hourly data for Eugene, Oregon.

Fig. 3. DIRINT hourly model errors for the DNI as a function of $\theta_{z}$ for Eugene, Oregon.

Fig. 4. GTI-DIRINT hourly model errors for the DNI as a function of $\theta_{z}$ and $\theta$ for Eugene, Oregon. Values of $\theta$ for the GTI from which the DNI and DHI were determined are denoted by color-coded symbols.

Fig. 5. GHI hourly errors when determined from GTI-DIRINT hourly values of DNI and DHI for Eugene, Oregon. Values of $\theta$ for the GTI from which the DNI and DHI were determined are denoted by color-coded symbols.

Fig. 6. MBDs for the Perez tilted surface model when modeling the GHI and GTIs using inputs of DNI and DHI from: measurements, DIRINT, and GTI-DIRINT.

Fig. 7. RMSDs for the Perez tilted surface model when modeling the GHI and GTIs and inputs of DNI and DHI from: measurements, DIRINT, and GTI-DIRINT. 


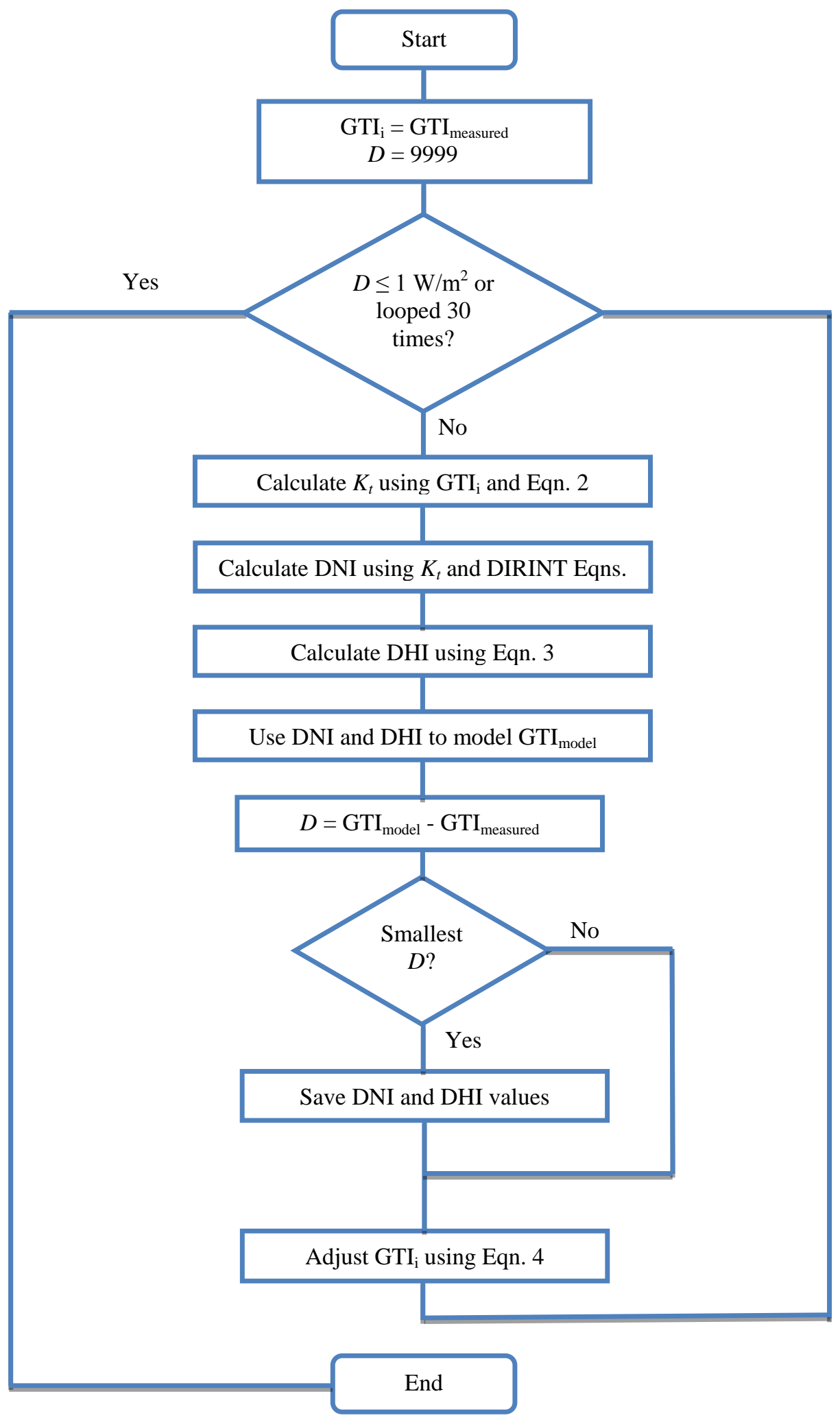




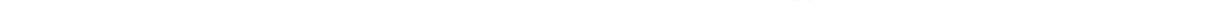




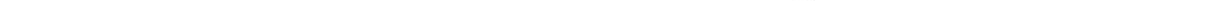




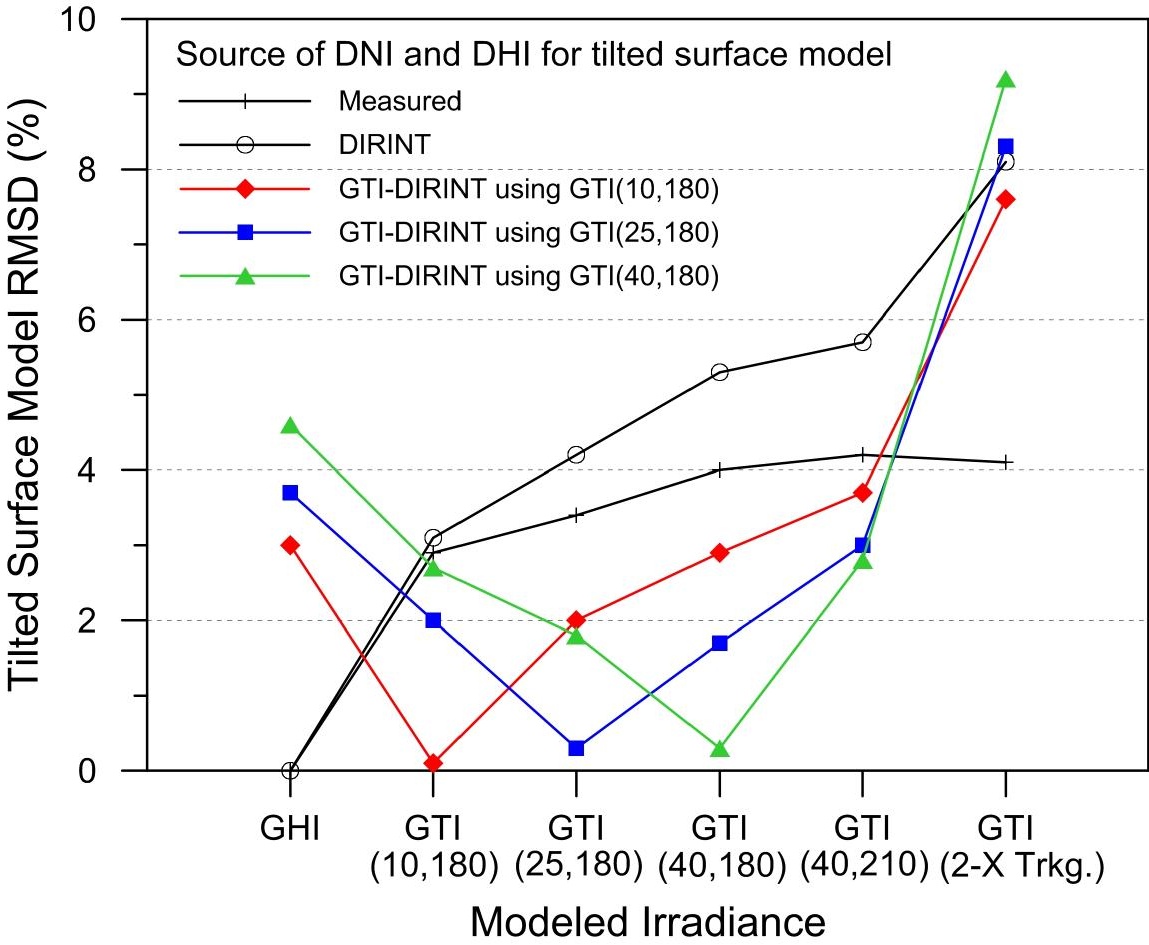


Table 1

Mean daytime irradiance values $\left(\mathrm{W} / \mathrm{m}^{2}\right)$

\begin{tabular}{llll}
\hline Irradiance & Cocoa, FL & Eugene, OR & Golden, CO \\
\hline DNI & 401 & 323 & 476 \\
DHI & 157 & 131 & 147 \\
GHI & 414 & 325 & 423 \\
GTI(28.5, 180) & 455 & & \\
GTI(44, 180) & & 353 & \\
GTI(10, 180) & & & 457 \\
GTI(25, 180) & & & 483 \\
GTI(40, 180) & & & 489 \\
GTI(40, 210) & & & 660 \\
GTI(2-X Trkg.) & & & \\
\hline
\end{tabular}

Table 2

Mean bias deviation (MBD) and root-mean-square deviation (RMSD) for DIRINT and GTI-DIRINT modeled values of DNI and DHI (using dew point temperature input) and for modeled GHI and GTI(latitude, 180) when using modeled and measured values of DNI and DHI for input to the tilted surface model

\begin{tabular}{|c|c|c|c|c|c|c|c|c|c|c|c|c|c|c|}
\hline \multirow[t]{2}{*}{ Location } & \multirow[t]{2}{*}{ Model/Method } & \multirow[t]{2}{*}{ Interval } & \multirow{2}{*}{$\begin{array}{r}\text { DNI } \\
\text { MBD } \\
(\%) \\
\end{array}$} & \multirow[b]{2}{*}{$\begin{array}{r}\text { RMSD } \\
(\%) \\
\end{array}$} & \multirow[b]{2}{*}{$\left(\mathrm{W} / \mathrm{m}^{2}\right)$} & \multirow{2}{*}{$\begin{array}{r}\mathrm{DHI} \\
\mathrm{MBD} \\
(\%) \\
\end{array}$} & \multirow[b]{2}{*}{$\begin{array}{c}\text { RMSD } \\
(\%)\end{array}$} & \multirow[b]{2}{*}{$\left(\mathrm{W} / \mathrm{m}^{2}\right)$} & \multirow{2}{*}{$\begin{array}{r}\text { GHI } \\
\text { MBD } \\
(\%) \\
\end{array}$} & \multirow[b]{2}{*}{$\begin{array}{c}\text { RMSD } \\
(\%)\end{array}$} & \multirow[b]{2}{*}{$\left(\mathrm{W} / \mathrm{m}^{2}\right)$} & \multicolumn{3}{|c|}{ GTI(latitude, 180) } \\
\hline & & & & & & & & & & & & $\begin{array}{r}\text { MBD } \\
(\%) \\
\end{array}$ & $\begin{array}{c}\text { RMSD } \\
(\%)\end{array}$ & $\left(\mathrm{W} / \mathrm{m}^{2}\right)$ \\
\hline \multirow[t]{9}{*}{ Cocoa, FL } & DIRINT & $5-\min$ & 3.7 & 21.8 & 88 & -5.0 & 37.1 & 58 & & & & -0.4 & 4.0 & 18 \\
\hline & & $15-\min$ & 2.3 & 20.8 & 84 & -3.0 & 34.6 & 55 & & & & -0.3 & 4.1 & 19 \\
\hline & & hour & -1.4 & 18.3 & 75 & 2.5 & 31.1 & 50 & & & & -0.5 & 3.2 & 15 \\
\hline & GTI-DIRINT & 5 -min & 5.1 & 22.9 & 92 & -4.8 & 37.9 & 59 & 0.5 & 3.8 & 16 & & & \\
\hline & & $15-\min$ & 2.2 & 22.7 & 91 & -1.6 & 35.6 & 56 & 0.3 & 4.0 & 17 & & & \\
\hline & & hour & 0.2 & 19.7 & 79 & 4.0 & 31.9 & 50 & 0.6 & 3.2 & 13 & & & \\
\hline & Meas. DNI, DHI & $5-\min$ & & & & & & & & & & -0.6 & 3.1 & 14 \\
\hline & & $15-\min$ & & & & & & & & & & -0.4 & 3.2 & 15 \\
\hline & & hour & & & & & & & & & & -0.4 & 2.2 & 11 \\
\hline \multirow[t]{9}{*}{ Eugene, OR } & DIRINT & $5-\min$ & 3.9 & 26.8 & 87 & -4.2 & 37.3 & 49 & & & & 1.4 & 7.3 & 26 \\
\hline & & $15-\min$ & 2.9 & 25.3 & 82 & -3.1 & 34.9 & 46 & & & & 1.2 & 6.9 & 24 \\
\hline & & hour & -0.4 & 21.7 & 71 & 1.1 & 29.0 & 38 & & & & 0.8 & 5.8 & 21 \\
\hline & GTI-DIRINT & $5-\min$ & 1.1 & 30.0 & 97 & -3.8 & 37.8 & 49 & -1.1 & 6.4 & 21 & & & \\
\hline & & $15-\min$ & -0.9 & 29.5 & 96 & -1.8 & 36.2 & 47 & -1.0 & 6.7 & 22 & & & \\
\hline & & hour & -4.0 & 28.0 & 91 & 3.8 & 31.8 & 42 & -0.9 & 4.9 & 16 & & & \\
\hline & Meas. DNI, DHI & $5-\min$ & & & & & & & & & & 0.0 & 4.5 & 16 \\
\hline & & 15-min & & & & & & & & & & 0.0 & 4.2 & 15 \\
\hline & & hour & & & & & & & & & & 0.3 & 3.4 & 12 \\
\hline \multirow[t]{9}{*}{ Golden, CO } & DIRINT & $5-\min$ & -1.8 & 19.5 & 93 & 1.7 & 36.2 & 53 & & & & -0.6 & 8.7 & 43 \\
\hline & & 15-min & -3.1 & 19.1 & 91 & 4.1 & 36.6 & 54 & & & & -0.2 & 6.8 & 33 \\
\hline & & hour & -5.8 & 19.5 & 93 & 10.6 & 37.3 & 55 & & & & -0.6 & 5.2 & 26 \\
\hline & GTI-DIRINT & $5-\min$ & -2.6 & 23.0 & 110 & 3.8 & 39.4 & 58 & 0.3 & 8.5 & 36 & & & \\
\hline & & $15-\min$ & -5.8 & 24.1 & 114 & 6.1 & 39.2 & 58 & -0.5 & 6.9 & 30 & & & \\
\hline & & hour & -6.2 & 20.7 & 99 & 13.1 & 40.4 & 59 & 0.3 & 4.7 & 20 & & & \\
\hline & Meas. DNI, DHI & $5-\min$ & & & & & & & & & & -0.1 & 7.9 & 39 \\
\hline & & $15-\min$ & & & & & & & & & & 0.3 & 5.8 & 29 \\
\hline & & hour & & & & & & & & & & 0.4 & 4.0 & 19 \\
\hline
\end{tabular}


Table 3

Mean bias deviation (MBD) and root-mean-square deviation (RMSD) for DIRINT and GTI-DIRINT modeled values of DNI and DHI (without using dew point temperature input) and for modeled GHI and GTI(latitude, 180) when using modeled and measured values of DNI and DHI for input to the tilted surface model

\begin{tabular}{|c|c|c|c|c|c|c|c|c|c|c|c|c|c|c|}
\hline \multirow[t]{2}{*}{ Location } & \multirow[t]{2}{*}{ Model/Method } & \multirow[t]{2}{*}{ Interval } & \multirow{2}{*}{$\begin{array}{r}\text { DNI } \\
\text { MBD } \\
(\%) \\
\end{array}$} & \multirow[b]{2}{*}{$\begin{array}{r}\text { RMSD } \\
(\%) \\
\end{array}$} & \multirow[b]{2}{*}{$\left(\mathrm{W} / \mathrm{m}^{2}\right)$} & \multirow{2}{*}{$\begin{array}{l}\text { DHI } \\
\text { MBD } \\
(\%) \\
\end{array}$} & \multirow[b]{2}{*}{$\begin{array}{c}\text { RMSD } \\
(\%) \\
\end{array}$} & \multirow[b]{2}{*}{$\left(\mathrm{W} / \mathrm{m}^{2}\right)$} & \multirow{2}{*}{$\begin{array}{l}\text { GHI } \\
\text { MBD } \\
(\%) \\
\end{array}$} & \multirow[b]{2}{*}{$\begin{array}{c}\text { RMSD } \\
(\%) \\
\end{array}$} & \multirow[b]{2}{*}{$\left(\mathrm{W} / \mathrm{m}^{2}\right)$} & \multicolumn{3}{|c|}{ GTI(latitude, 180) } \\
\hline & & & & & & & & & & & & $\begin{array}{r}\text { MBD } \\
(\%) \\
\end{array}$ & $\begin{array}{c}\text { RMSD } \\
(\%) \\
\end{array}$ & $\left(\mathrm{W} / \mathrm{m}^{2}\right)$ \\
\hline \multirow[t]{9}{*}{ Cocoa, FL } & DIRINT & $5-\min$ & 3.7 & 20.5 & 83 & -4.5 & 34.0 & 53 & & & & -0.2 & 4.0 & 18 \\
\hline & & $15-\min$ & 1.5 & 19.8 & 80 & -1.1 & 32.4 & 51 & & & & -0.1 & 4.0 & 18 \\
\hline & & hour & -3.1 & 17.8 & 73 & 5.5 & 30.1 & 48 & & & & -0.4 & 3.1 & 14 \\
\hline & GTI-DIRINT & $5-\min$ & 5.4 & 23.0 & 92 & -4.5 & 33.7 & 53 & 0.4 & 3.9 & 16 & & & \\
\hline & & $15-\min$ & 1.8 & 22.7 & 91 & -0.1 & 32.6 & 51 & 0.1 & 3.9 & 16 & & & \\
\hline & & hour & -0.8 & 20.3 & 82 & 6.9 & 30.4 & 48 & 0.6 & 3.3 & 14 & & & \\
\hline & Meas. DNI, DHI & $5-\min$ & & & & & & & & & & -0.6 & 3.1 & 14 \\
\hline & & $15-\min$ & & & & & & & & & & -0.4 & 3.2 & 15 \\
\hline & & hour & & & & & & & & & & -0.4 & 2.2 & 11 \\
\hline \multirow[t]{9}{*}{ Eugene, OR } & DIRINT & $5-\min$ & 6.8 & 27.6 & 89 & -7.8 & 37.8 & 49 & & & & 1.7 & 7.5 & 27 \\
\hline & & $15-\min$ & 5.8 & 25.9 & 84 & -6.7 & 35.3 & 46 & & & & 1.6 & 7.1 & 25 \\
\hline & & hour & 1.9 & 21.8 & 72 & -1.9 & 28.9 & 38 & & & & 1.2 & 6.0 & 21 \\
\hline & GTI-DIRINT & $5-\min$ & 4.2 & 29.9 & 97 & -7.7 & 37.4 & 49 & -1.1 & 6.4 & 21 & & & \\
\hline & & 15-min & 1.9 & 29.2 & 95 & -5.6 & 35.2 & 46 & -1.2 & 6.7 & 22 & & & \\
\hline & & hour & -1.4 & 26.2 & 85 & 0.5 & 29.8 & 39 & -0.9 & 4.9 & 16 & & & \\
\hline & Meas. DNI, DHI & $5-\min$ & & & & & & & & & & 0.0 & 4.5 & 16 \\
\hline & & $15-\min$ & & & & & & & & & & 0.0 & 4.2 & 15 \\
\hline & & hour & & & & & & & & & & 0.3 & 3.4 & 12 \\
\hline \multirow[t]{9}{*}{ Golden, CO } & DIRINT & $5-\min$ & 2.0 & 19.2 & 92 & -5.1 & 35.1 & 52 & & & & 0.2 & 8.7 & 43 \\
\hline & & $15-\min$ & 0.5 & 18.5 & 89 & -2.7 & 34.5 & 51 & & & & 0.6 & 6.8 & 33 \\
\hline & & hour & -2.5 & 18.6 & 89 & 3.8 & 34.1 & 50 & & & & 0.2 & 5.2 & 25 \\
\hline & GTI-DIRINT & $5-\min$ & 1.1 & 22.5 & 107 & -3.8 & 36.8 & 54 & -0.1 & 8.4 & 36 & & & \\
\hline & & $15-\min$ & -2.3 & 22.9 & 109 & -1.2 & 36.0 & 53 & -0.8 & 6.8 & 29 & & & \\
\hline & & hour & -3.2 & 19.3 & 92 & 6.2 & 35.6 & 52 & -0.1 & 4.5 & 19 & & & \\
\hline & Meas. DNI, DHI & $5-\min$ & & & & & & & & & & -0.1 & 7.9 & 39 \\
\hline & & $15-\min$ & & & & & & & & & & 0.3 & 5.8 & 29 \\
\hline & & hour & & & & & & & & & & 0.4 & 4.0 & 19 \\
\hline
\end{tabular}

Table 4

Mean bias deviation (MBD) and root-mean-square deviation (RMSD) for DIRINT and GTI-DIRINT modeled values of DNI and DHI when using the GTI input for the various tilt and azimuth orientations for Golden, $\mathrm{CO}$ and for an hourly data averaging interval

\begin{tabular}{lrrrrrr}
\hline Model/Input & \multicolumn{1}{c}{ DNI } & \multicolumn{5}{c}{ DHI } \\
& $\begin{array}{r}\text { MBD } \\
(\%)\end{array}$ & $\begin{array}{r}\text { RMSD } \\
(\%)\end{array}$ & $\left(\mathrm{W} / \mathrm{m}^{2}\right)$ & $\begin{array}{r}\text { MBD } \\
(\%)\end{array}$ & $\begin{array}{r}\text { RMSD } \\
(\%)\end{array}$ & $\left(\mathrm{W} / \mathrm{m}^{2}\right)$ \\
\hline DIRINT & -2.5 & 18.6 & 89 & 3.8 & 34.1 & 50 \\
GTI-DIRINT & & & & & & \\
GTI(10, 180) & -2.7 & 18.2 & 88 & 4.5 & 34.5 & 51 \\
GTI(25, 180) & -4.2 & 18.4 & 88 & 5.2 & 34.5 & 51 \\
GTI(40, 180) & -3.2 & 19.3 & 92 & 6.2 & 35.6 & 52 \\
GTI(40, 210) & -6.6 & 35.0 & 166 & 10.6 & 53.3 & 78 \\
\hline
\end{tabular}

